\title{
Chitra Ultraviolet-C-Based Facemask Disposal Bin
}

\author{
Subhash Neyyattinkara Neelakandan ${ }^{1}$ (D) Aneesh Sukesan ${ }^{1} \cdot$ Jijo Jerard $^{1} \cdot$ Vivek Parayath Uthaman $^{1}$. \\ Vipin Dev Vasudevan ${ }^{1}$. Pradeep Kumar Soman Pillai Sarojini Amma ${ }^{1}$ Maya A. Nandkumar ${ }^{1}$. \\ Muraleedharan Chirathodi Vayalappil ${ }^{1}$
}

Received: 27 April 2020 / Revised: 9 June 2020 / Accepted: 13 June 2020 / Published online: 18 June 2020

(C) Indian National Academy of Engineering 2020

\begin{abstract}
Used face masks are hazardous waste and must be discarded immediately upon removal. Instead of throwing used masks into the disposal bin, disinfecting the masks is essential to break the chain of infection spread. The development of this device was attempted for COVID-19 management, with the following focus: (1) solution which have sufficient science background established so that extensive experimental validation need not be attempted during the lockdown period in India. (2) Provide solution which could be replicated with local resources and minimum material movement. Therefore, in response to the pressing societal demand we designed, prototyped, verified and validated an UV-C-based multipurpose disinfection device. The device after safety and efficacy evaluation as per the Indian Council of Medical Research (ICMR) guidelines for novel COVID-19 solutions, received Central Drugs Control Standard Organization (CDSCO) registration as non-notified medical device for commercialization.
\end{abstract}

Keywords UV-C $\cdot$ Disinfection $\cdot$ Facemask $\cdot$ Disposal

\section{Introduction}

Waste management personnel are at the risk of COVID-19 exposure while performing their duties. Proper disinfection and disposal of used facemask is of paramount importance in breaking the chain to prevent the virus spread. Hence it is recommended that public and health workers should dispose their used facemasks in a hygienic way instead of randomly throwing them into waste bin together with other waste. Numerous thermal, chemical and radiation disinfection methods have been developed over the past: among these, ultraviolet C (UV-C) germicidal disinfection method deploys UV-C radiation to disinfect microorganisms (Hijnen et al. 2006; She et al. 2020).

In the electromagnetic spectrum, UV light falls between visible light and $\mathrm{X}$-rays. UV light is categorized as: Far UV from 100 to $200 \mathrm{~nm}$, UV-C from 200 to $280 \mathrm{~nm}$, UV-B from 280 to $315 \mathrm{~nm}$, and UV-A from 315 to $400 \mathrm{~nm}$ (Hijnen

Subhash Neyyattinkara Neelakandan

subhashnn@sctimst.ac.in

1 Biomedical Technology Wing, Sree Chitra Tirunal Institute for Medical Sciences and Technology, Trivandrum 695012, India et al. 2006; She et al. 2020; Malayeri et al. 2016). Previous research has shown that when microorganisms are exposed to UV-C, the absorption by DNA or RNA prevents cell multiplication and reproduction (Hijnen et al. 2006; She et al. 2020; Malayeri et al. 2016).

Microorganism like bacteria consists of one cell which contains deoxyribonucleic acid (DNA) whereas viruses are smaller than bacteria and are enclosed by a protein coat (CIE 2003). Viruses contains one or more molecules of DNA or Ribonucleic acid (RNA). UV-C exposure results in the destruction of nucleic acid present in microorganisms through induction of hydrates, uracil and thymine dimers (Beck et al. 2016; CDC 2008). Hence UV-C absorption by a microorganism's RNA and DNA led to photochemical destruction. This results in prevention of replication and subsequent inactivation of the microorganisms. This is the mechanism of UV-C disinfection of bacteria, viruses, fungi and protozoa. The UV-C-induced damaged occurs at UV-C wavelength (200-280 nm) absorbed by RNA and DNA. Germicidal lamps generate $85 \%$ of their radiant output near Ultraviolet-C $254 \mathrm{~nm}$, which is closer to the peak germicidal efficacy (CIE 2003). SARS-CoV-2, a type of corona virus, are RNA viruses enveloped with a lipid bilayer. Lipid viruses are least resistant to germicidal disinfection 
compared to Bacterial Spores, Mycobacteria, Non-lipid or small Viruses, Fungi and Vegetative Bacteria (FDA 2020).

As per World Health Organization interim guidance on 15 May 2020, the solutions used for disinfection should be discarded after each use. The buckets used must be washed, rinsed, dried and stored in inverted position when not in use. Personnel preparing and using chemical disinfectants should use specific personal protective equipment due to high concentration, and longer exposure to the chemical disinfectants during the work hours (WHO 2020). In addition, the operating cost of the UV-C disinfection device includes only annual lamp replacement and energy consumption. UV-C-based disinfection device eliminates the cost and risk associated with transport, storage and delivery of chemical disinfectants. Operator or user training is comparatively easy in UV-C disinfection device when compared with chemical disinfection. Collectively these factors, and no chemical toxicity concerns, give UV-C-based disinfection device better utility for disinfection of used facemasks before disposal. Also, ICMR has designated Sree Chitra Tirunal Institute for Medical Sciences and Technology (SCTIMST) for the evaluation of UV-C-based COVID-19 solutions in India prior to commercialization (ICMR 2020).

This study and device development was undertaken with the following focus: (1) Solution which have sufficient science background established so that extensive experimental validation need not be attempted during the lockdown. (2) Provide solution which could be replicated with local resources (like metallic casing, UV-C light lamp and fixture, timer circuit and inner reflective surfaces) and minimum material movement. Considering design for manufacturability, procurement and cost, this study investigates the relation between (1) distance from light source, (2) number of light lamps and (3) reflective inner surfaces, on the UV-C dose distribution inside the UV-C bin system. The efficacy of the UV-C-based facemask disposal bin is validated by microbiological studies as per ICMR guidelines for COVID19 solutions.

\section{Methods}

\section{Design Risk Analysis and Management}

Occupational Health and Safety Officials (OSHA) has provided the guidance for ultraviolet radiation exposure (NIOSH 1972). Over exposure of UV-C may led to skin irritation and photo-conjunctivitis. Hence, eye and skin exposure time limit the usage of UV-C during human occupancy of design spaces. The threshold limit values (TLV) recommended by American Conference of Governmental Industrial Hygienists (ACGIH) for $253.7 \mathrm{~nm} \mathrm{UV-C} \mathrm{is} 6 \mathrm{~mJ} /$ $\mathrm{cm}^{2}$ for any 8-h period (NIOSH 1972, 1973).
So, the risk mitigation strategy adopted during UV-C disinfection device design was:

a. Human exposure must be avoided while the UV-C light is switched ON.

b. If the user accidently opens the door during disinfection process, the UV-C light will be switched off.

c. UV-C stable metallic enclosure made of stainless steel, mild steel or aluminum should be used for the construction of device.

d. The design space, the dimensions of the UV-C disinfection device, and UV-C lamp specification was arrived in such a way that minimum required UV-C energy per unit area is ensured at all spatial points inside.

e. The reflective surfaces inside should ensure that there are no shadow areas or corners.

f. The efficacy of method is defined by the energy delivered per unit area. Sufficient safety margin should be provided in the delivered energy density level for mitigating the risk.

g. Instructions for use (IFU) and UV-C warning message/ symbol on product/package/brochure during commercialization.

\section{Chitra UV-C-Based Facemask Disposal Bin System}

Figure 1 shows the system level breakdown and Fig. 2 shows the exploded view of Chitra UV-C-based face mask disposal system used for evaluation of safety and efficacy. The design consisted of an Aluminum metallic bin (height $44 \mathrm{~cm}$, width $53.2 \mathrm{~cm}$ and depth $43 \mathrm{~cm}), 15 \mathrm{~W}$ UV-C lamp (M/s. SANKYODENKI G15T8) of $254 \mathrm{~nm}$ with holder accessories, and aluminum foils as internal reflective surfaces. A timer circuit (Fig. 3) which detects the door closure and switches on the UV-C lamp after a delay of 3-10 s was also provided. The circuit consists of a limit switch signal, attached to the input of microcontroller's digital pin and the signal was fed to timer circuit logic block. Timer circuit logic block generate appropriate delay for the UV-C lamp and the resultant signal was fed to relay module block. The function of relay module was to Turn ON and Turn OFF of the UV-C lamp. The indicating LED's status were: (1) Green LED: Glows when the disinfection device is ready to use. (2) Yellow LED: Glows when the UV-C device door was open. (3) Red LED: Glows when the UV-C lamp was ON. The electronic controls were designed to switch OFF the lamp either after 3 min of exposure or during accidental opening of the door.

\section{Design of Experiments (DOE) for Manufacturability}

DoE was used to develop a transfer function to determine the effect of control factors (distance from the lamp, number of lamps, reflective surface) on UV-C dose inside the 
Fig. 1 System to component level breakdown

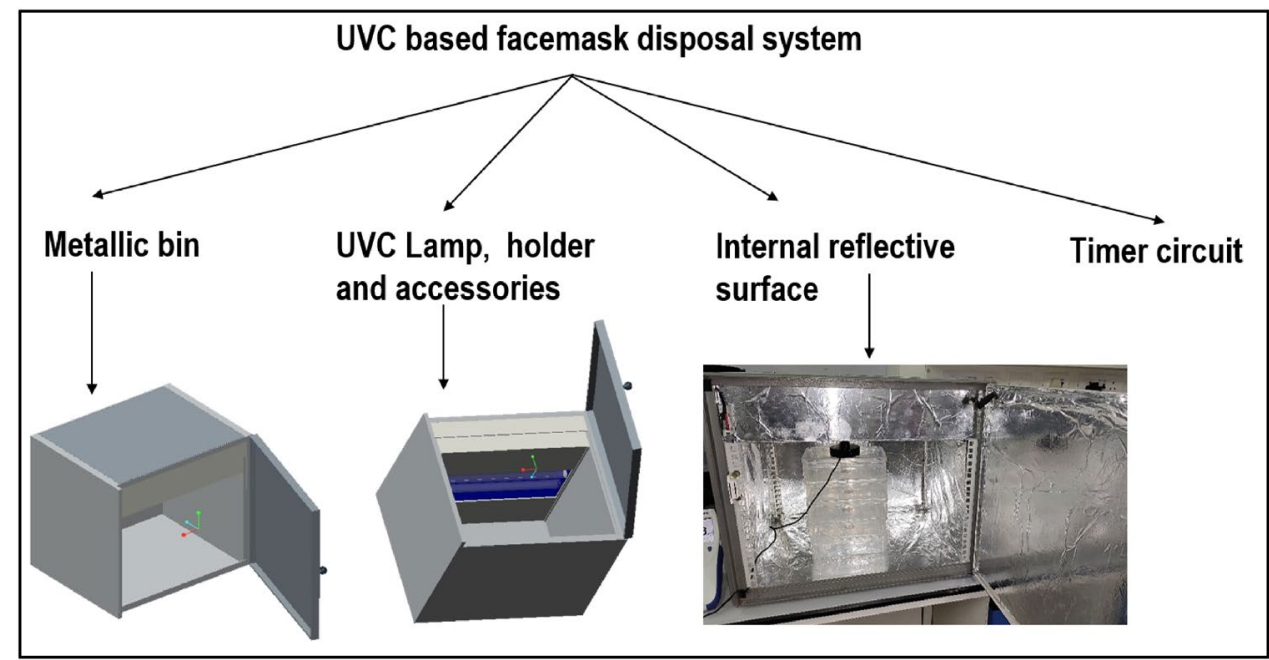

Fig. 2 Exploded view of Chitra UV-C-based facemask disposal bin

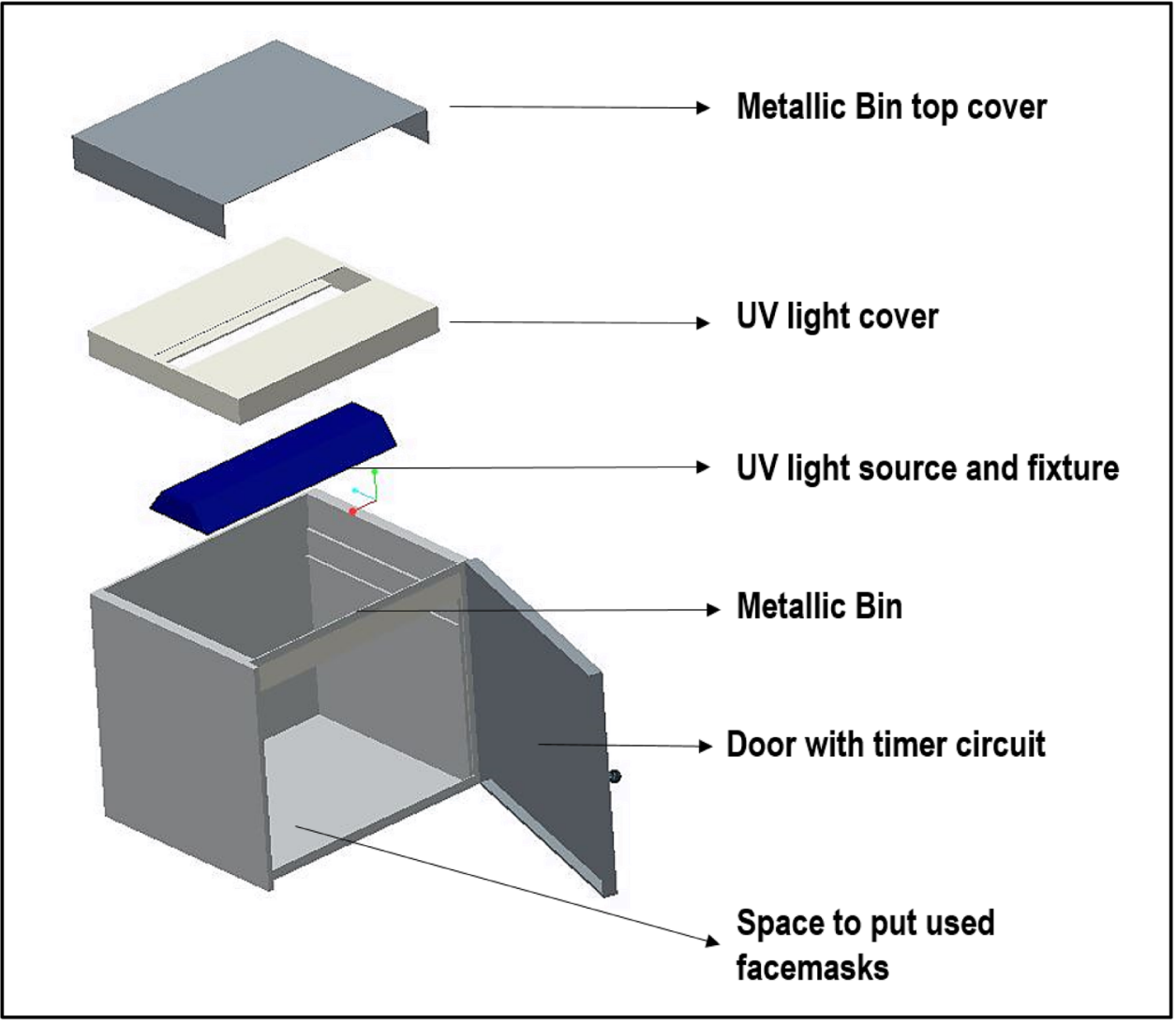

design space. The DoE analysis was essential considering Design for Manufacturability and cost consideration under present lock down scenario. The control factors shown in Table 1: (1) distance from light source, (2) no. of light lamps and (3) reflective inner surfaces were varied systematically to understand the UV-C dose inside the bin. Full factor DOE was carried out for all three factors (Fig. 4) and total eight combinations were tested.

\section{Device Validation: Efficacy Evaluation}

The organisms used in the study were S. aureus ATCC 25,923, E.coli ATCC 25,922, Pseudomonas aeruginosa ATCC 9027, Bacillus subtilis ATCC 6633, Candia albicans ATCC 10,231. The microbial culture of an inoculum density of about $10^{4} \mathrm{cfu} / \mathrm{ml}$ was inoculated on agar plates by spread plating. The culture plate was placed inside the 


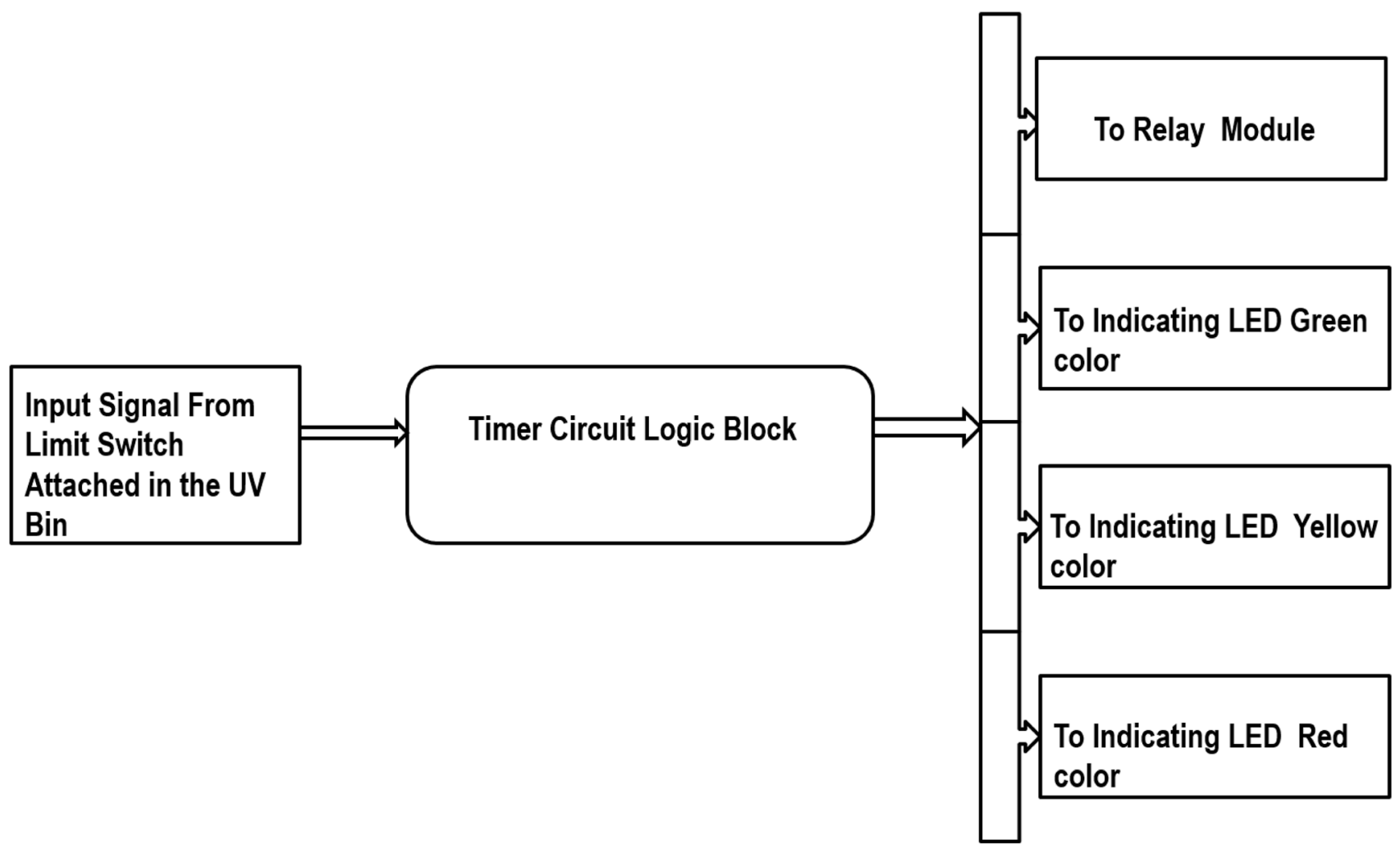

Fig. 3 Block diagram of timer circuit

Table 1 Control factors

\begin{tabular}{llll}
\hline Sl. no & Factor & Lower limit & Upper limit \\
\hline 1 & $\begin{array}{c}A=\text { Distance from lamp } \\
(\mathrm{cm})\end{array}$ & 4.6 & 31.5 \\
2 & $B=$ No of lamps & 1 lamp & 2 lamps \\
3 & $C=$ Reflective surface & No reflective surface & $\begin{array}{c}\text { Al foil } \\
\text { reflective } \\
\text { surfaces }\end{array}$ \\
\hline
\end{tabular}

Design of experiments (DOE) for manufacturability

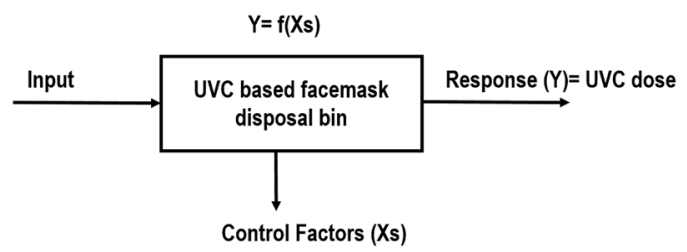

Fig. 4 P diagram
Table 2 UV-C dose $\left(\mathrm{mJ} / \mathrm{cm}^{2}\right)$ for disinfection

\begin{tabular}{llll}
\hline Sl. no & Microorganisms & $3 \log$ reduction & $\begin{array}{l}4 \log \\
\text { reduc- } \\
\text { tion }\end{array}$ \\
\hline 1 & S. aureus ATCC 25,923 & 6.5 & 10 \\
2 & E.Coli ATCC 25,922 & 7 & 8 \\
3 & $\begin{array}{l}\text { Pseudomonas aeruginosa ATCC } \\
\text { 9027 }\end{array}$ & 10 & 17 \\
4 & Bacillus subtilis ATCC 6633 & 61 & 82 \\
\hline
\end{tabular}

UV-C disinfection space and was exposed to UV-C as given below.

1. Exposed to UV for $3 \mathrm{~min}$ with one $15 \mathrm{~W}$ UV-C lamp ON.

2. Exposed to UV for 10 minutes with one $15 \mathrm{~W} \mathrm{UV}-\mathrm{C}$ lamp ON.

3. Exposed to UV for 3 min with two $15 \mathrm{~W}$ UV-C lamps ON.

4. Exposed to UV for 10 min with two $15 \mathrm{~W}$ UV-C lamps ON. 
After UV exposure the agar plates are incubated and examined for microbial growth. Non-virus strain (Table 2) was used for validation because of Biosafety Level 3 (BSL3) requirements (Mourya et al. 2014). The UV-C dose required for disinfection based on previous research was shown in Table 2 (Malayeri et al. 2016). The chosen nonvirus strains for validation had similar UV-C resistance as that of most of the viruses (Malayeri et al. 2016). Also SARS-CoV-2, a type of corona viruses, are least resistant to germicidal disinfection compared to Bacterial Spores, Mycobacteria, Non-lipid or small Viruses, Fungi and Vegetative Bacteria (FDA 2020).

\section{Results}

\section{Design Verification: UV-C dose Measurement}

The UV-C dose of the bin was measured using a power meter (Newport Model 843R). Significant increase in the UV-C dose was observed while internal reflective surfaces were used (Fig. 5). Also increasing the number of UV-C lamps resulted in higher UV-C dose (Fig. 6).

\section{Design of Experiments}

UV-C dose measured for the full factorial DOE is shown in Table 3.

The reduced model was developed from the initial full factorial model by removing only one model term at a time with (1) Coefficients with $p$ value $>0.05$, (2) starting with complex interaction terms, (3) replicating run till all coefficients have $p$ value less than 0.5 (3) residual normality check to determine fit errors, (4) pattern analysis to determine whether the data is under normal random category to ensure usability of transfer function. Hence the final transfer function was derived with all significant terms.

$Y_{\mathrm{UVC} \text { dose }}=f(A, B, C)$,

UVC dose of bin $=133.07-2.3196 A+0.49 B-1.39 C$

$$
+0.7679 A B+0.3541 A C
$$

where $A$ is the distance from lamp in $\mathrm{cm}, B$ is the number of lamps, $C$ is the reflective surface, are the main effects and; $\mathrm{AB}$ and $\mathrm{AC}$ are the interaction effects. The above relation can be effectively used for making data driven design decisions while prototyping the UV-C disinfection device.

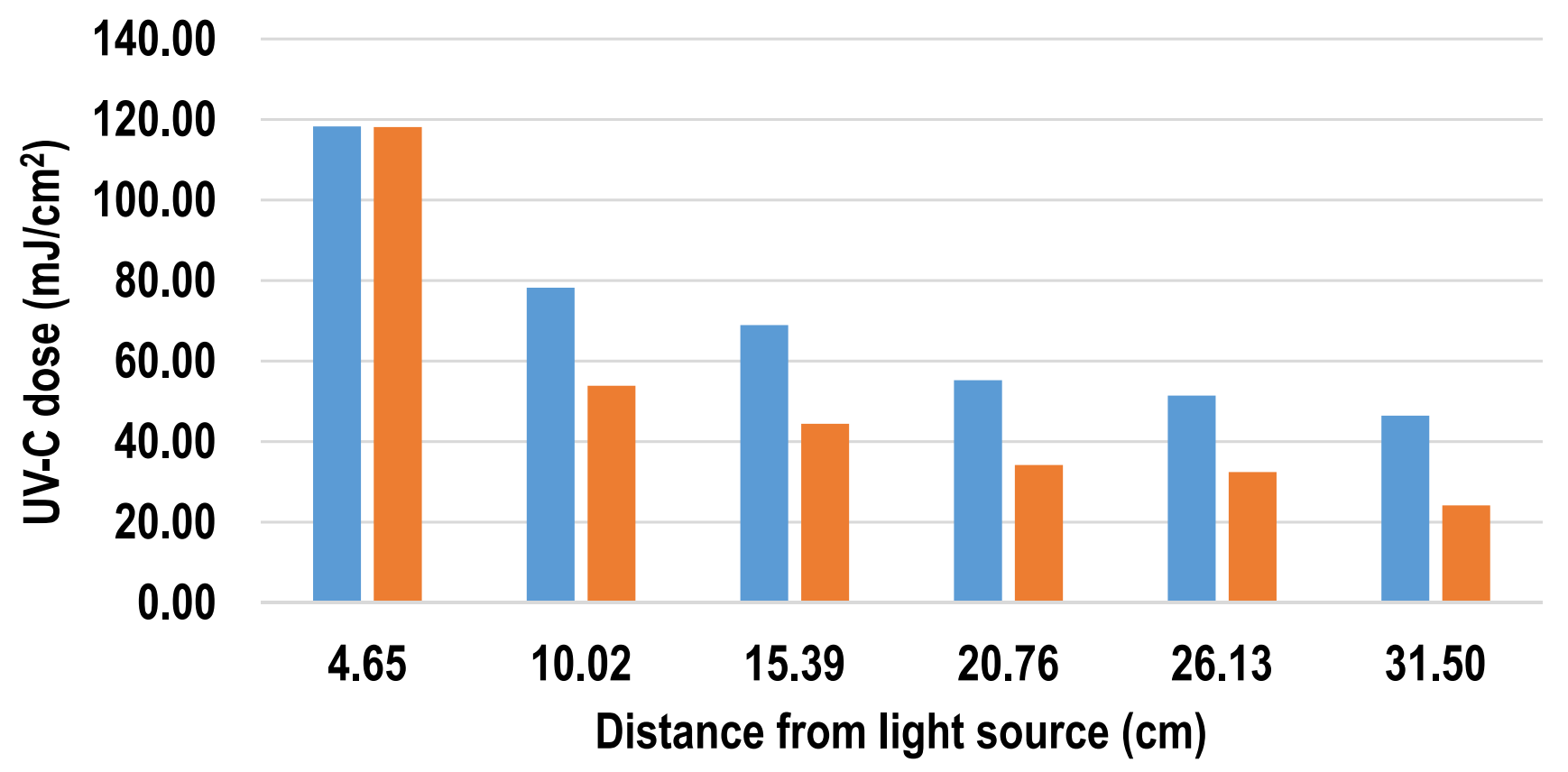

UV-C dose with reflector

Fig. 5 Measured UV-C dose of bin with one lamp for 1-min exposure 


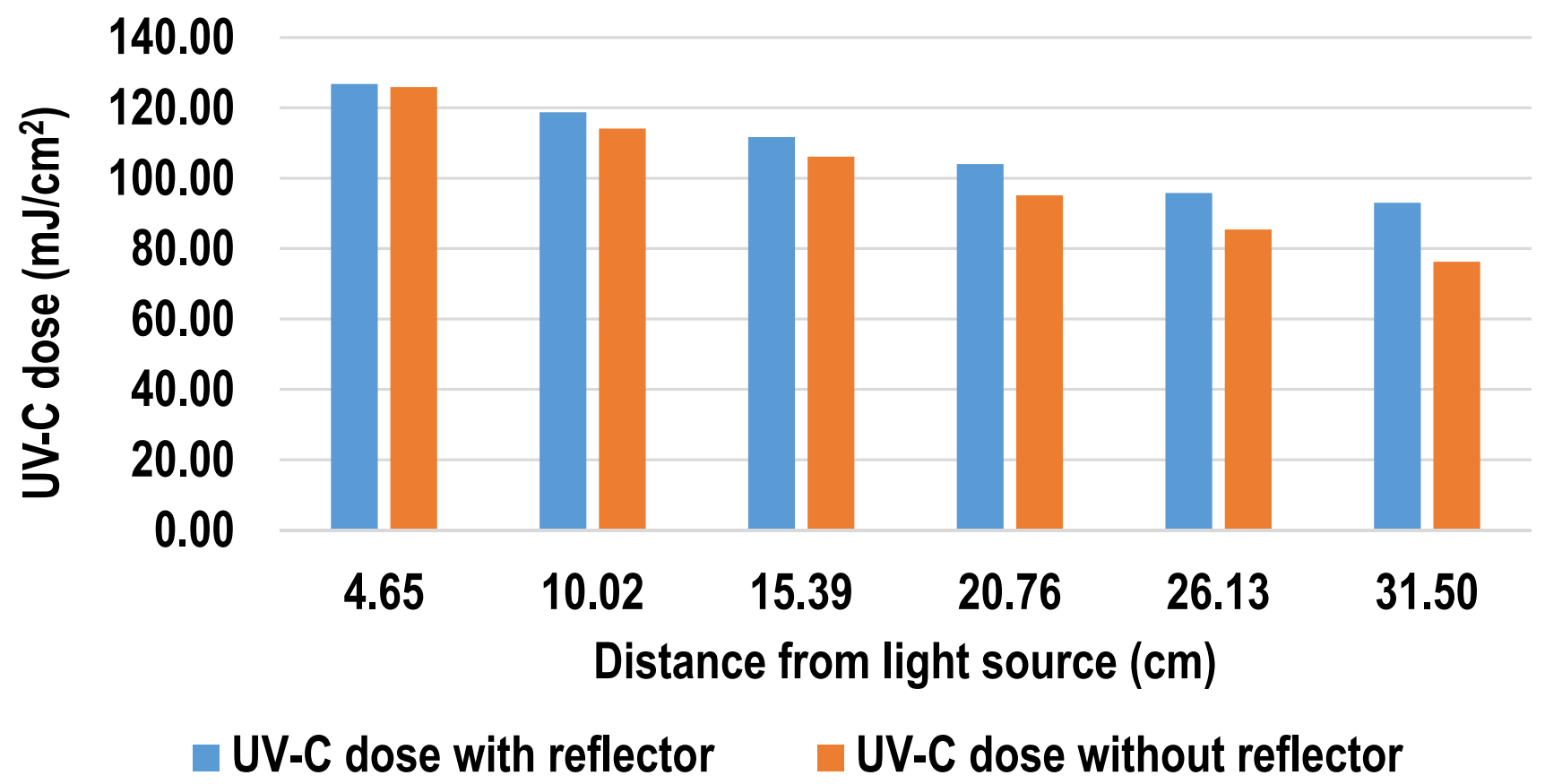

Fig. 6 Measured UV-C dose of bin with two lamps for 1-min exposure

Table 3 DOE results

\begin{tabular}{lcllc}
\hline Sl. no & $\begin{array}{l}\text { Distance from lamp } \\
(\mathrm{cm})\end{array}$ & No. of lamps & Reflective surface & $\begin{array}{l}\text { UV-C dose } \\
\left(\mathrm{mJ} / \mathrm{cm}^{2}\right)\end{array}$ \\
\hline 1 & 4.65 & 2 Bulbs & Reflective surfaces & 126.76 \\
2 & 31.50 & 1 Bulb & Reflective surfaces & 46.48 \\
3 & 4.65 & 1 Bulb & Reflective surfaces & 118.31 \\
4 & 4.65 & 2 Bulbs & No reflective surface & 125.92 \\
5 & 31.50 & 1 Bulb & No reflective surface & 24.17 \\
6 & 4.65 & 1 Bulb & No reflective surface & 118.14 \\
7 & 31.50 & 2 Bulbs & Reflective surfaces & 93.04 \\
8 & 31.50 & 2 Bulbs & No reflective surface & 76.31 \\
\hline
\end{tabular}

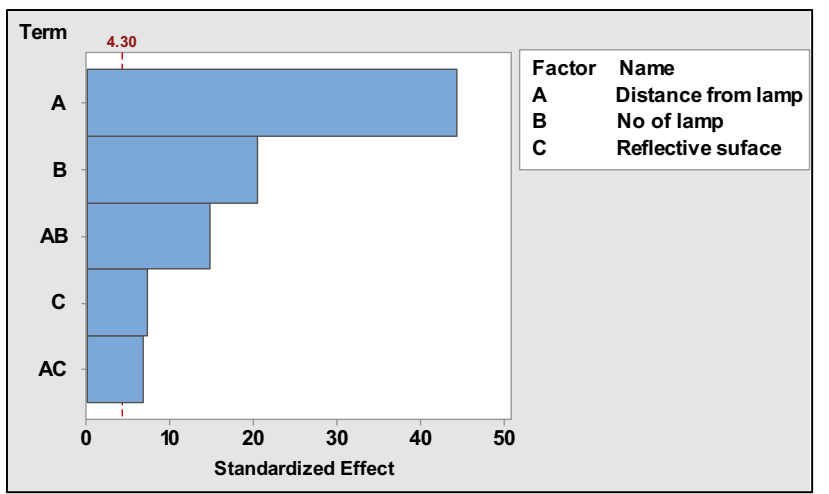

Fig. 7 Ranking of factors affecting UV-C dose
The factors were ranked based on the coefficient of the reduced model of transfer function as shown in the Pareto chart from Minitab (Fig. 7).

\section{Device Validation: Efficacy Evaluation}

On UV-C exposure for 3 min itself, using two $15 \mathrm{~W}$ UV-C lamps, there was $4 \log$ reduction in the microorganisms tested. The experiment was repeated using one $15 \mathrm{~W}$ UV-C lamp and similar observation was made. 


\section{Discussion}

\section{Design of Experiments}

Distance from the lamp has most significant impact on the device function, followed by number of lamps. When an interaction effect is present between the control factors ( $A, B$ and $C$ ), the output function or response of the device changes upon the level of mutual interaction. It was observed that for same distance the drop of UV-C dose for two lamps was less when compared with one lamp. So, the factors distance from the lamp $(A)$ and number of lamps $(B)$ has significant mutual interaction $(A B)$. Based on the coefficients of the reduced model of transfer function the mutual interaction $A B$ is most significant followed by $A C$.

\section{Multipurpose of UV-C Disinfection Device}

Firstly, the UV-C-based disinfection device can be used for multipurpose disinfection of used facemasks, gloves, and PPE items prior to disposal. But the disinfection for PPE and gloves were not validated in the present study. It should be noted that the efficacy of the UV-C disinfection depends on the line of sight effect of the radiation. So, the efficacy of disinfection of folded PPE and gloves should be validated prior to use and commercialization. Secondly, the same device can be used for disinfection of UV-C stable metallic objects like those in cutlery, beauty and saloons, as metals are stable under UV-C exposure. If there is no sufficient data to address the potential risk of material degradation and compromise in functionality of the non-metallic devices kept inside, UV-C-based disinfection is not recommended. The efficacy of method is defined by the energy delivered per unit area. The condition of the mask (like soiled, dirty) may have marginal effects on efficacy. Sufficient safety margin, two UV-C lamps instead one, has been provided in the device to deliver energy density level for mitigating the risk.

\section{Fast Track Commercialization During COVID-19 and Regulatory Pathway}

The devices developed by us has two commercial variants. First, the used face mask is inserted one at a time (Figs. 8, 9). After disinfection, the mask from the disinfection device is automatically transferred to the waste bin below. Second, the used face masks when arranged in one row without overlapping, results in $4 \log$ reduction of microbes (Fig. 10). The variant Bin19, technology know how by SCTIMST and manufactured by VST Mobility solutions has the following features:

1. When the user waves hand in front of the bin, it is shutter automatically opens.

2. After putting the used face mask, one at a time, the shutter closes automatically. Followed by that he disinfection process begins. After the disinfection the mask will be automatically transferred from the disinfection space, to a waste bin below. Both the disinfection space and waste bin is enclosed by a metallic enclosure.

3. The user can disinfect hands, with the help of an integrated auto dispensing sanitizer.

4. Once the waste bin is full of facemasks, ready for disposal, the IOT enabled Bin19 send message to the maintenance team for waste removal.

Timeline from ideation to commercialization readiness was 8 weeks, which consists of: (1) One week from ideation to proof-of -concept (POC) stage, (2) Safety and efficacy validation as per ICMR guidelines of novel COVID-19 solutions at SCTIMST in next 2 weeks, (3) Technology transfer to industry partners through expression of interest, (4) 2-3 weeks for Central Drugs Standard Control Organization (CDSCO) registration after necessary certifications for Quality Management Systems for the industry partner, (5) Parallel, in a fast track mode, procurement and production

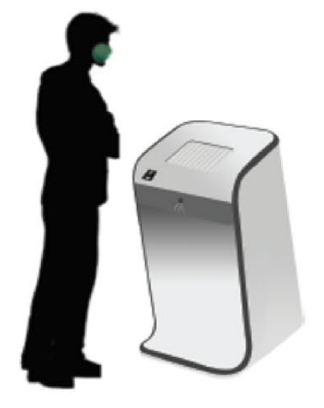

Bin19
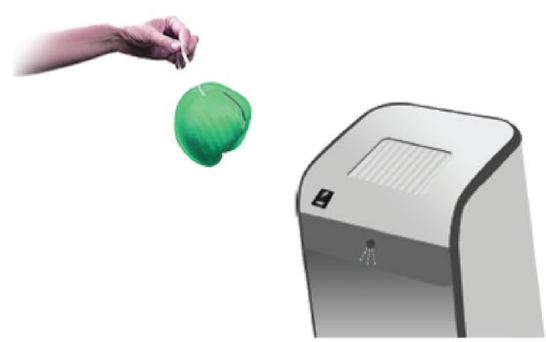

Shutter automatically opens

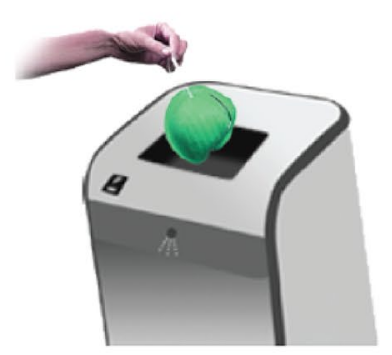

Disposing mask one at a time

Fig. 8 Bin19 operation 


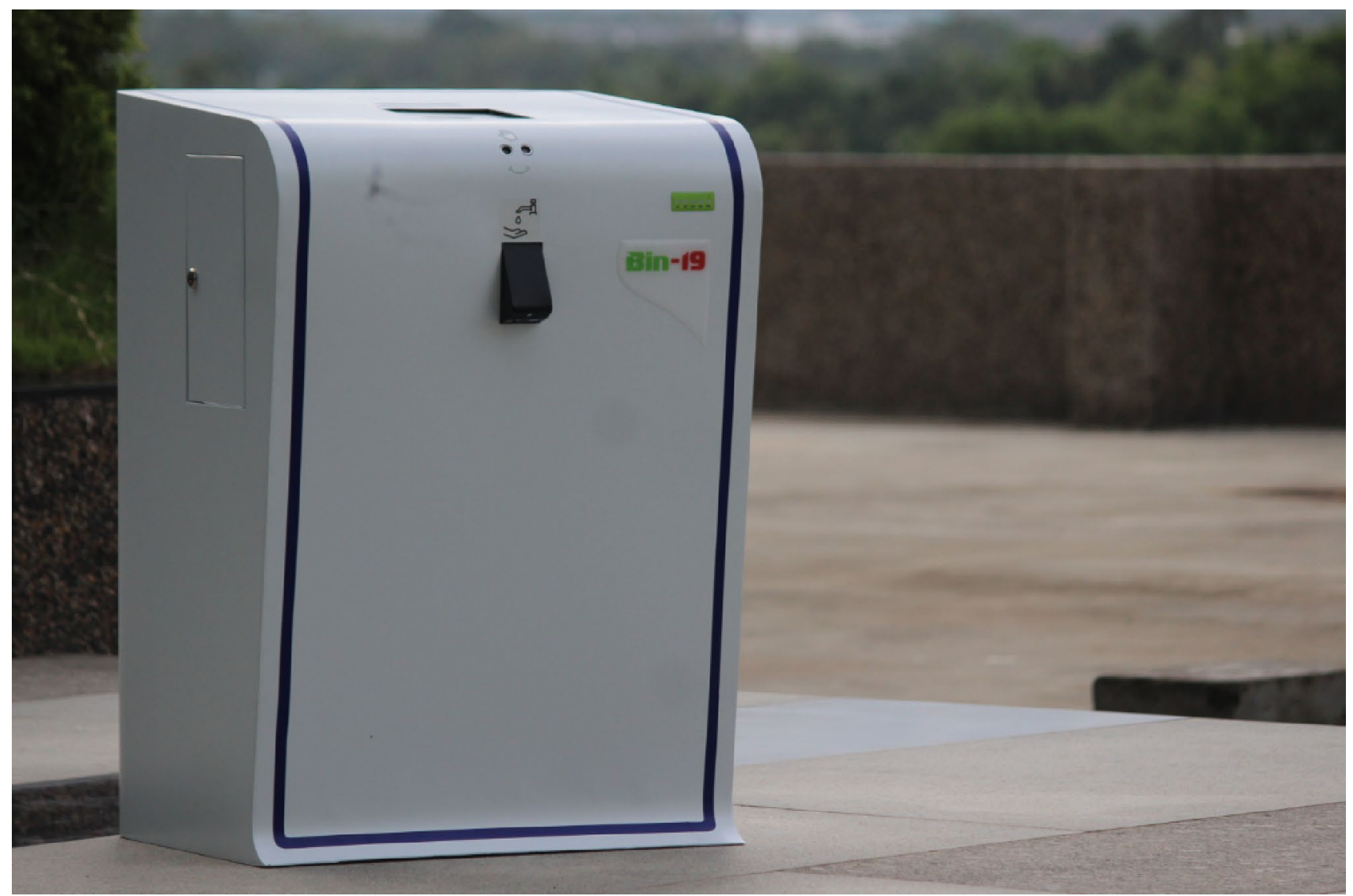

Fig. 9 Product Bin19: Developed and transferred by SCTIMST, manufactured by VST Mobility Solutions, and registered under Central Drugs Standard Control Organization, Govt. of India

Fig. 10 Product UV Spot: Developed and transferred by SCTIMST, manufactured by VST Mobility Solutions, and registered under Central Drugs Standard Control Organization, Govt. of India

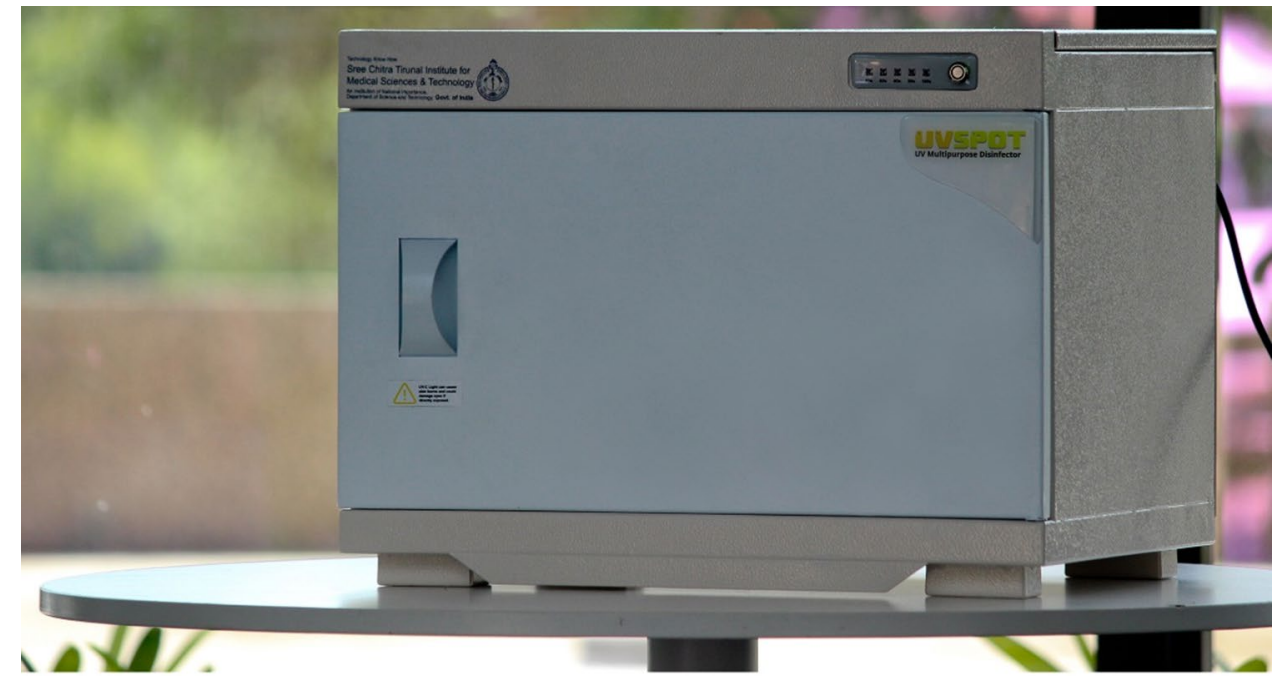


scale up from the industry took another 2 weeks due to lockdown. However, SCTIMST is a designated center for evaluation of novel COVID-19 solutions as per ICMR guidelines, the safety and efficacy evaluation by SCTIMST fast-tracked the ideation to commercialization readiness.

\section{Conclusions}

The results of the study bring out the following inferences:

1. Ultraviolet-C bin with internal reflective surfaces was tested for safety and efficacy. 4 log reduction in microorganisms tested were observed on UV-C exposure for three minutes, using one $15 \mathrm{~W}, 254 \mathrm{~nm}$, UV-C lamp.

2. The device can be used for disinfection of used facemasks before disposal, thereby reducing the risk of waste collectors from being exposed to hazardous waste. Also the multipurpose disinfector can be used for disinfection of UV-C stable metallic objects.

3. The device could be replicated with local resources and minimum material movement.

4. The UV-C disinfection innovations during COVID-19 in India must be evaluated for safety and efficacy as per ICMR guidelines. SCTIMST, being a designated center for regulate and evaluate the UV-C-based COVID-19 solutions, accelerated the technology translation to commercialization.

Acknowledgements This work was supported by many divisions/ departments of the Sree Chitra Tirunal Institute for Medical Sciences and Technology, Trivandrum. The device was transferred to multiple industry partners (VST Mobility Solutions, PMG ISD, Vivesty Green etc.) through expression of interest for commercialization. This work was supported by Technical Research Centre (TRC) for Biomedical Devices, Department of Science and Technology (DST), Govt. of India

\section{References}

Beck SE, Rodriguez RA, Hawkins MA, Hargy TM, Larason TC, Linden KG (2016) Comparison of UV-induced inactivation and RNA damage in MS2 phage across the germicidal UV spectrum. Appl Environ Microbiol 82(5):1468-1474
Centers for Disease Control and Prevention (CDC) (2008) Guideline for disinfection and sterilization in healthcare facilities. https:// www.cdc.gov/infectioncontrol/guidelines/disinfection/index.html. Accessed June 2, 2020.

Chang JC, Ossoff SF, Lobe DC, Dorfman MH, Dumais CM, Qualls RG, Johnson JD (1985) UV inactivation of pathogenic and indicator microorganisms. Appl Environ Microbiol 49(6):1361-1365

Hijnen WA, Beerendonk EF, Medema GJ (2006) Inactivation credit of UV radiation for viruses, bacteria and protozoan (oo) cysts in water: a review. Water Res 40(1):3-22

Indian Council of Medical research (ICMR) (2020) Guidance for evaluation of novel applications for COVID-19, ICMR, Department of Health Research. Accessed 30 May 2020.

International Commission on Illumination (CIE) (2003) CIE 155:2003 ultraviolet air disinfection. https://files.cie.co.at/cie15 5-2003\%2520(free\%2520copy\%2520March\%25202020).pdf. Accessed 2 Jun 2020.

Malayeri AH, Mohseni M, Cairns B, Bolton JR, Chevrefils G, Caron E, Barbeau B, Wright H, Linden KG (2016) Fluence (UV dose) required to achieve incremental log inactivation of bacteria, protozoa, viruses and algae. IUVA News 18(3):4-6

Mourya DT, Yadav PD, Majumdar TD, Chauhan DS, Katoch VM (2014) Establishment of biosafety level-3 (BSL-3) laboratory: important criteria to consider while designing, constructing, commissioning and operating the facility in Indian setting. Indian $\mathbf{J}$ Med Res 140(2): 171

NIOSH (1972) Criteria for a recommended standard: occupational exposure to ultraviolet radiation, DHHS (NIOSH) Publication Number 73-11009.

NIOSH (1973) Ultraviolet radiation and the work environment, national institute for occupational safety and health, DHHS (NIOSH) Publication Number 73-11005.

She RC, Chen D, Pak P, Armani DK, Schubert A, Armani AM (2020) Build-at-home UV-C disinfection system for healthcare settings. arXiv preprint arXiv:2003.12916

US Food and Drug Administration (FDA) (2020) Enforcement policy for sterilizers, disinfectant devices, and air purifiers during the coronavirus disease 2019 (COVID-19) Public Health Emergency, Guidance for Industry and Food and Drug Administration Staff. https://www.fda.gov/regulatory-information/search-fda-guida nce-documents/enforcement-policy-sterilizers-disinfectant-devic es-and-air-purifiers-during-coronavirus-disease. Accessed 2 Jun 2020.

World Health Organization (2020) Cleaning and disinfection of environmental surfaces in the context of COVID-19, Interim guidance, 15 May 2020. World Health Organization.

Publisher's Note Springer Nature remains neutral with regard to jurisdictional claims in published maps and institutional affiliations. 